\title{
ALTERACIONES AUDITIVAS EN TRABAJADORES EXPUESTOS AL RUIDO INDUSTRIAL
}

\author{
ADEL HERNÁNDEZ DIAZ*, \\ BIANKA M. GONZÁLEZ MÉNDEZ** \\ (*) Especialista en Medicina Familiar. \\ Centro de Aplicaciones Tecnológicas y Desarrollo Nuclear (CEADEN) \\ (**) Especialista en Medicina Familiar \\ Policlínico Universitario "Luis Pasteur"
}

\section{RESUMEN}

Motivados por la importancia del ruido como riesgo laboral en las actividades productivas de la Carpintería de Aluminio "Tomás Alvarez Breto" decidimos realizar un control audiométrico periódico de estos trabajadores con la finalidad de determinar el grado de exposición de los mismos y establecer las recomendaciones más importantes para su protección. Nos propusimos como objetivo determinar el grado de afectación auditiva por ruido en los trabajadores expuestos al riesgo, para lo cual se cuantificaron los niveles de ruido existentes en los diferentes puestos de trabajo, se confeccionaron las historias clínicas y se realizó un examen otoscópico y una prueba audiométrica en ambos oídos para definir el daño acústico y la presencia de hipoacusia profesional en los obreros estudiados. Al concluir el estudio pudimos comprobar que el ruido constituía un contaminante de gran importancia en esta industria. Este riesgo laboral se encontraba por encima del nivel de seguridad de $85 \mathrm{db}(\mathrm{A})$ en 9 de los 13 departamentos con que cuenta el centro y ha afectado la salud de los trabajadores ya que existían 77 casos $(78,5 \%)$ de hipoacusia atribuible al ruido y un gran número de trabajadores $(30,6 \%)$ expuestos a elevados niveles de ruido innecesariamente por la naturaleza de su labor. Por todo esto recomendamos tomar medidas que reduzcan el nivel de ruido en los puestos de trabajo donde existían niveles superiores a los permitidos, exigirse el uso de los medios de protección auditiva en los trabajadores expuestos y cumplirse estrictamente con los exámenes médicos preventivos, incluyendo las pruebas audiométricas anualmente.

\section{PALABRAS CLAVES}

Hipoacusia profesional, ruido industrial, medios de protección

\section{ABSTRACT}

Motivated by the importance of the noise like labour risk in the productive activities of Aluminium Carpentry Tomas Alvarez Breto we decide to carry out a control periodic audiometric of these workers with the purpose of to determine the degree of exhibition of the same ones and to establish the most important recommendations for their protection. We intended as objective to determine the degree of auditory affectation for noise in the exposed workers to the risk, for that which the existent levels of noise were quantified in the different work positions, the clinical histories were made and he/she was carried out an exam otoscópico and a test audiometric in both hearings to define the acoustic damage and the presence of professional hipoacusia in the studied workers. When concluding the study we could check that the noise constituted a pollutant of great importance in this industry. This labour risk was above the level of security of $85 \mathrm{db}(\mathrm{A})$ in 9 of the 13 departments with which it counts the center and it has affected the health of the workers since 77 cases they existed $(78.5 \%)$ of attributable hipoacusia to the noise and a great number of workers $(30.6 \%)$ exposed at high levels of noise unnecessarily for the nature of its work. For all this we recommend to take immediate measures that reduce the level of noise in the work positions where superior levels existed to those allowed, the use of the means of acoustic protection should be demanded in the exposed workers and it should be fulfilled the preventive medical exams strictly, including the tests audiometric annually.

\section{KEY WORDS}

Professional hipoacusia, industrial noise, protection means. 


\section{INTRODUCCION}

El ruido se ha descrito como un sonido sin calidad musical agradable o como un sonido no querido o no deseado. No todos los sonidos son detectables por el oído humano ${ }^{1}$.

Este es sin lugar a dudas el primer factor contaminante que ha sido denunciado por la humanidad. En el siglo IV antes de Jesucristo ya se dictaban normas conducentes a reducir los niveles sonoros producidos por los artesanos y canteros ${ }^{2}$.

Las primeras referencias específicas sobre el daño a la audición humana causada por ruido se encuentran recogidas en el Régimen Sanitatis Salerenitanum que fue escrito en el año 1150 de nuestra era, lo que hace pensar que los efectos nocivos del ruido ya eran conocidos desde la época en que la industria existente era sólo artesanal ${ }^{3}$.

En 1713 Bernardo Ramazzini escribió en su obra "De Morbis Artificium Diatriba" que los trabajadores del cobre perdían la audición a causa del martillo sobre el metal, motivo por el cual, aquellas personas que llegan a viejo haciendo el mismo trabajo expuestos a ruido, terminarían siendo sordas por completo ${ }^{4}$.

El ruido como riesgo laboral es conocido desde hace muchos años. Sin embargo, no es hasta el advenimiento de la revolución industrial, cuando las fábricas comienzan a sustituir la fuerza humana por máquinas, que cobra verdaderamente importancia la exposición a ruido como factor de producción de alteraciones de la salud en los trabajadores ${ }^{5}$.

La exposición corta a ruido excesivo por encima de los $85 \mathrm{db}(\mathrm{A})$ origina primero un desplazamiento temporal del umbral de audibilidad (DTU) conocido por período de fatiga auditiva que desaparece después de algunos minutos u horas de reposo. A medida que aumenta el tiempo de exposición o la intensidad, o se suman ambos factores, el desplazamiento del umbral aumenta y la recuperación de la audición no tiene lugar a los niveles iniciales. En esta fase, la pérdida auditiva residual se denomina desplazamiento permanente del umbral de audibilidad (DPU) o hipoacusia causada por el ruido, caracterizada por comenzar en las frecuencias alrededor de los $4000 \mathrm{~Hz}$. Al principio se desarrolla una sordera sin signos clínicos que sólo se evidencia por la audiometría y no afecta las frecuencias conversacionales. Al final se produce una ampliación del déficit auditivo en la zona conversacional que es socialmente perjudicial ${ }^{6.7 .8}$.
El efecto nocivo del ruido sobre la agudeza auditiva del individuo depende de varios factores tales como: frecuencia, pureza, intensidad, duración o tiempo de exposición, repetición, edad del trabajador y susceptibilidad individual ${ }^{1.8 .9}$.

El ruido no sólo ocasiona alteraciones en el aparato auditivo, también actúa sobre los centros bulbares, vegetativos, centros corticales de asociación y de la voluntad. Está considerado entre los factores que predisponen a la fatiga mental y física, que suelen reflejarse en tasas más elevadas de ausentismo y de inestabilidad del personal. Además puede obstaculizar la comunicación hablada, molestar y distraer, reducir el rendimiento y la eficacia, aparte de varios trastornos en la salud que no guardan relación con los efectos auditivos ${ }^{1.10}$.

Los estímulos sonoros fuertes producen una vasoconstricción a distintos niveles periféricos, además de disminuir la capacidad de reacción del individuo y su rendimiento laboral. Se ha demostrado que en fábricas similares, las de mayor índices de accidentes laborales con aquellas que presentan mayores niveles de ruido ${ }^{11.12}$.

Como resultado de la exposición a niveles elevados de ruido industrial se produce hipoacusia o sordera profesional que no es más que la pérdida de la audición de ambos oídos, irreversible y acumulativa de tipo nervioso sensoneural que afecta las frecuencias conversacionales ${ }^{1.2 .13}$.

La importancia de este contaminante ambiental ha motivado que se hayan realizado numerosas investigaciones acerca de este tema, lo que ha permitido en los últimos años un mayor conocimiento y establecimiento de normales y recomendaciones por diferentes países y organismos ${ }^{14}$.

Motivados por la importancia de este riesgo laboral en las actividades de la Carpintería de Aluminio "Tomás Alvarez Breto" decidimos realizar un control audiométrico periódico de esos trabajadores, con la finalidad de determinar el grado de exposición de los mismos y establecer las recomendaciones más importantes para su protección.

\section{OBJETIVOS}

\section{General}

- Determinar el grado de afectación auditiva por ruido en los trabajadores expuestos al ruido industrial. 


\section{Especificos}

1. Cuantificar los niveles de ruido existentes en los diferentes puestos de trabajo y departamentos de este centro.

2. Definir el daño acústico de cada trabajador mediante el resultado del audiograma, relacionándolo con la edad, sexo, tiempo de exposición y ocupación.

3. Demostrar la presencia de hipoacusia profesional en los obreros estudiados.

\section{MATERIAL Y METODO}

Se llevó a cabo un estudio descriptivo-retrospectivo del universo de 98 trabajadores de la Carpintería de Aluminio "Tomás Alvarez Breto", situada en la Ciudad de La Habana; la cual se dedicaba al desarrollo y producción de la marquetería de aluminio (puertas, ventanas y barandas) destinada a instituciones estatales.

Esta investigación comprendió los siguientes aspectos:

- Se realizaron determinaciones de los niveles de ruido en $\mathrm{db}(\mathrm{A})$ en cada uno de los puntos establecidos en los locales de trabajo y departamentos del centro cumpliendo con los requisitos establecidos ${ }^{18}$.

- A cada trabajador se le confeccionó una Historia Clínica donde se recogieron los siguientes datos:

- Nombre

- Edad

- Sexo

- Puesto de trabajo

- Tiempo de exposición al ruido

- Uso de los medios de protección auditiva

- Antecedentes patológicos personales (APP) y familiares (APF)

- Posteriormente se le realizó a cada obrero un examen otoscópico y una prueba audiométrica en ambos oídos. La audiometría por vía ósea se efectuó solo en los casos que presentaron déficit marcado de la audición o llevaban mayor tiempo laborando en ambiente ruidoso.

La prueba consistió en examinar el oído en las frecuencias comprendidas entre 0.5-6 Khz., dándo- sele primero la señal de la frecuencia y después partiendo de cero, se fue incrementando el estímulo sonoro en intervalos de $5 \mathrm{db}$ hasta que el trabajador percibiera el sonido; seguidamente se hizo lo mismo con el otro oído.

Los audiogramas fueron realizados en una Cabina de Sonotex, con un nivel de ruido de fondo inferior a los $35 \mathrm{db}(\mathrm{A})$, lo cual cumple con lo establecido por la mayoría de los autores (I.L.O.) ${ }^{19}$.

Se utilizó el modelo 62-02 para audiometrías y se le exigió a cada trabajador como mínimo 16 horas sin exposición al ruido.

El criterio diagnóstico se basó en encontrar un escotoma en la frecuencia de $4 \mathrm{Khz}$. que según se profundiza y toma las frecuencias de 2 y $3 \mathrm{Khz}$. (teniendo en cuenta la pérdida producida por la edad) determina la gravedad ${ }^{13,20,21}$.

El vaciamiento de los datos se realizó en un modelo de recolección confeccionado al efecto y posteriormente se procesó toda la información utilizando el método estadístico descriptivo.

La información está presentada en forma de gráficos y tablas.

\section{ANALISIS DE LOS RESULTADOS:}

A través del análisis de la Tabla I podemos conocer que en la encuesta realizada se obtuvo un promedio de edad de 39,1 años; con un máximo de edad de 59 años y un mínimo de 21 años.

El grupo etáreo donde se encontró el mayor número de obreros fue el de 26 a 35 años, lo que representó el $38,9 \%$ del total estudiado.

Con relación al sexo el grupo femenino fue el más numeroso correspondiendo al $55,0 \%$ del universo analizado.

La Tabla II nos muestra la distribución de los trabajadores por departamentos y tiempo de exposición al ruido.

La edad laboral promedio encontrada fue de 13.9 años con un máximo de 41 años laborados y un mínimo de 6 meses.

El mayor número de trabajadores se encontró en los grupos entre 1 y 5 años y entre 6 y 10 años de labor para un $28,0 \%$. 
Tabla I. Universo de trabajadores por edad y sexo

\begin{tabular}{|c|c|c|c|c|c|c|}
\hline \multirow{3}{*}{$\begin{array}{c}\text { GRUPOS DE } \\
\text { EDADES }\end{array}$} & \multicolumn{4}{|c|}{ SEXO } & & \\
\hline & \multicolumn{2}{|c|}{ MASCULINO } & \multicolumn{2}{|c|}{ FEMENINO } & \multicolumn{2}{|c|}{ TOTAL } \\
\hline & \# & $\%$ & $\#$ & $\%$ & \# & $\%$ \\
\hline 25 años o menos & 7 & 7.1 & - & - & 7 & 7.1 \\
\hline 26-35 años & 13 & 13.3 & 25 & 25.6 & 38 & 38.9 \\
\hline 36-45 años & 7 & 7.1 & 16 & 16.3 & 23 & 23.4 \\
\hline 46-55 años & 10 & 10.2 & 11 & 11.3 & 21 & 21.5 \\
\hline 56 años o más & 7 & 7.1 & 2 & 2.0 & 9 & 9.1 \\
\hline TOTAL & 44 & 44.8 & 54 & 55.0 & 98 & 100.0 \\
\hline
\end{tabular}

Fuente: Dpto. de Recursos Humanos Carpintería de Aluminio

Tabla II. Universo de trabajadores por departamentos y tiempo de exposición

\begin{tabular}{|c|c|c|c|c|c|c|c|c|c|c|c|c|c|c|c|c|}
\hline \multirow{3}{*}{$\begin{array}{l}\text { DEPARTA- } \\
\text { MENTOS }\end{array}$} & \multicolumn{14}{|c|}{ TIEMPO DE EXPOSICIÓN AL RUIDO } & \multirow{2}{*}{\multicolumn{2}{|c|}{ TOTAL }} \\
\hline & \multicolumn{2}{|c|}{$<1 \mathrm{ANO}$} & \multicolumn{2}{|c|}{ 1-5 ANOS } & \multicolumn{2}{|c|}{ 6-10ANOS } & \multicolumn{2}{|c|}{ 11-15 ANOS } & \multicolumn{2}{|c|}{ 16-20 ANOS } & \multicolumn{2}{|c|}{ 21-30 ANOS } & \multicolumn{2}{|c|}{$>31$ ANOS } & & \\
\hline & $\#$ & $\%$ & \# & $\%$ & $\#$ & $\%$ & \# & $\%$ & $\#$ & $\%$ & $\#$ & $\%$ & $\#$ & $\%$ & \# & $\%$ \\
\hline $\begin{array}{l}\text { AREA } \\
\text { SIERRAS }\end{array}$ & - & - & - & - & 1 & 1.0 & 1 & 1.0 & - & - & - & - & - & - & 2 & 2.0 \\
\hline BARANDA & 1 & 1.0 & 2 & 2.0 & 1 & 1.0 & 1 & 1.0 & 1 & 1.0 & 1 & 1.0 & - & - & 7 & 7.0 \\
\hline V.M.Q & - & - & - & - & 3 & 3.2 & 1 & 1.0 & 2 & 2.0 & 1 & 1.0 & - & - & 7 & 7.2 \\
\hline V.T.A. & - & - & 2 & 2.0 & 3 & 3.2 & - & - & 2 & 2.0 & - & - & - & - & 7 & 7.2 \\
\hline $\begin{array}{l}\text { ACCE- } \\
\text { SORIOS }\end{array}$ & - & - & 4 & 4.2 & 1 & 1.0 & 1 & 1.0 & 1 & 1.0 & - & - & - & - & 7 & 7.2 \\
\hline $\begin{array}{l}\text { MANTENI- } \\
\text { MIENTO }\end{array}$ & 1 & 1.0 & 4 & 4.2 & 1 & 1.0 & - & - & 2 & 2.0 & 1 & 1.0 & 1 & 1.0 & 10 & 10.2 \\
\hline $\begin{array}{l}\text { DPTO } \\
\text { TECNICO }\end{array}$ & - & - & 1 & 1.0 & 2 & 2.0 & - & - & - & - & - & - & - & - & 3 & 3.0 \\
\hline PUERTA & - & - & 1 & 1.0 & 5 & 5.2 & 1 & 1.0 & 2 & 2.0 & 2 & 2.0 & 2 & 2.0 & 13 & 13.2 \\
\hline RECEPCIÓN & - & - & 1 & 1.0 & - & - & 1 & 1.0 & 1 & 1.0 & - & - & - & - & 3 & 3.0 \\
\hline OFICINAS & - & - & 4 & 4.2 & 4 & 4.2 & 2 & 2.0 & 2 & 2.0 & - & - & - & - & 12 & 12.4 \\
\hline ALMACENN & - & - & - & - & 1 & 1.0 & - & - & 1 & 1.0 & - & - & - & - & 2 & 2.0 \\
\hline $\begin{array}{l}\text { PLANTA } \\
\text { ANODIZADO }\end{array}$ & - & - & 3 & 3.2 & 2 & 2.0 & 1 & 1.0 & 2 & 2.0 & 3 & 3.2 & - & - & 11 & 11.4 \\
\hline $\begin{array}{l}\text { COCINA-- } \\
\text { COMEDOR }\end{array}$ & - & - & 1 & 1.0 & 1 & 1.0 & - & - & 2 & 2.0 & 2 & 2.0 & - & - & 6 & 6.0 \\
\hline $\begin{array}{l}\text { ESTIBA- } \\
\text { DORES }\end{array}$ & 2 & 2.0 & 4 & 4.2 & 2 & 2.0 & - & - & - & - & - & - & - & - & 6 & 6.2 \\
\hline TOTAL & 4 & 4.0 & 27 & 28.0 & 27 & 28.0 & 9 & 9.0 & 18 & 18.0 & 10 & 10.2 & 3 & 3.0 & 98.0 & 100.0 \\
\hline
\end{tabular}

Fuente: Dpto. de Recursos Humanos Carpintería de Aluminio 
En cuanto a la utilización de los medios de protección auditiva no existía ningún trabajador que utilizara los mismos durante la jornada laboral.

En la Tabla III encontramos las mediciones de los niveles de ruido realizadas en los 13 locales de trabajo del centro donde nos preocupa que, solo en 4 de ellos los mismos fueran iguales o inferiores a 85 $\mathrm{db}(\mathrm{A})$, que es el límite máximo admisible para 8 horas diarias de exposición sin que ocasione una afectación auditiva.

En las 9 áreas restantes los trabajadores se encontraban expuestos a niveles sonoros que pueden causar daños a su salud.

Como puede observarse, el departamento de Baranda resultó el de mayor nivel sonoro con valores de hasta $101 \mathrm{db}(\mathrm{A})$. Es de resaltar el Área de Sierras donde se encontraron niveles entre 104 y 107 $\mathrm{db}(\mathrm{A})$ y por la cual rotan trabajadores de diferentes departamentos.

Los estibadores se mantenían fundamentalmente en las áreas exteriores, alejados de los talleres, por lo que no se exponían al riesgo laboral.

El Gráfico I refleja que de todos los trabajadores estudiados sólo 21 presentaron resultados audiomé- tricos normales $(21,5 \%)$, mientras que los 77 restantes arrojaron hipoacusia $(78,5 \%)$.

En la Tabla IV podemos apreciar como los departamentos con mayor porciento de trabajadores afectados fueron Puerta y Mantenimiento que se corresponden con los niveles sonoros más elevados.

Es de señalar que la Planta de Anodizado, a pesar de tener límites permisibles $83 \mathrm{db}(\mathrm{A})$, presentó un $9,2 \%$ de trabajadores con alteraciones auditivas. Esto se corresponde, al igual que en otros departamentos, a que en años anteriores los trabajadores rotaban periódicamente por los diferentes puestos de trabajo de la fábrica.

La Tabla V demuestra que el grupo de edades más aquejado fue el de 26 a 35 años, siguiéndole en orden de frecuencia los grupos de 36 a 45 y de 46 a 55 años con igual número de afectados.

Es de destacar que en el grupo de mayores de 56 años todos los obreros están afectados por el ruido.

En la Tabla VI encontramos que con relación al sexo de disminución de la capacidad auditiva fue mayor en las mujeres $(45.9 \%)$ que en los hombres $(32,7 \%)$.

Tabla III. Mediciones del nivel de ruido por departamentos

\begin{tabular}{|l|c|}
\hline \multicolumn{1}{|c|}{ DEPARTAMENTOS } & NIVEL SONORO db (A) \\
\hline 1. Área de Sierras & $104-107$ \\
\hline 2. Barandas & $87-101$ \\
\hline 3. VMQ (Marquesinas) & $87-98$ \\
\hline 4. VTA (Tablillas) & $87-97$ \\
\hline 5. Accesorios & $86-97$ \\
\hline 6. Mantenimiento & 92 \\
\hline 7. Dpto. Técnico & 89 \\
\hline 8. Puerta & $85-89$ \\
\hline 9. Recepción & 86 \\
\hline 10. Oficinas & 85 \\
\hline 11. Almacén de Ferretería & 85 \\
\hline 12. Planta de Anodizado & 83 \\
\hline 13. Cocina-Comedor & 83 \\
\hline 14. Estibadores & - \\
\hline
\end{tabular}

Fuente: Mediciones realizadas por medicina del trabajo 
Gráfico 1. Distribución de la muestra según resultados del audiograma

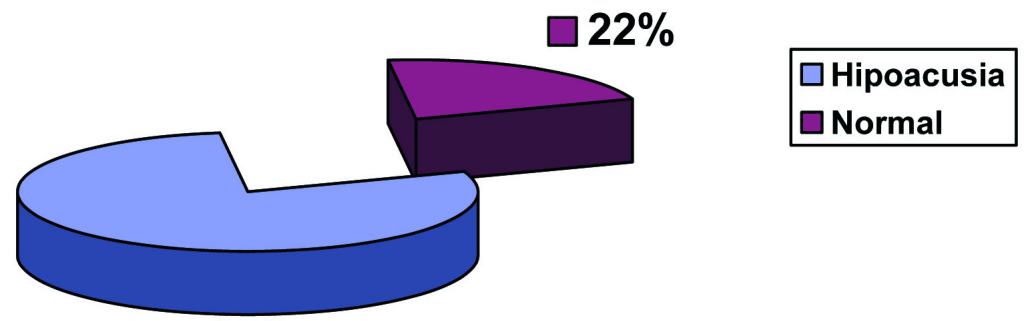

$78 \%$

Tabla IV. Resultados del audiograma por departamentos

\begin{tabular}{|c|c|c|c|c|c|c|}
\hline \multirow{3}{*}{ DEPARTAMENTOS } & \multicolumn{4}{|c|}{ RESULTADOS AUDIOGRAMA } & \multirow{2}{*}{\multicolumn{2}{|c|}{ TOTAL }} \\
\hline & \multicolumn{2}{|c|}{ NORMAL } & \multicolumn{2}{|c|}{ HIPOACUSIA } & & \\
\hline & \# & $\%$ & \# & $\%$ & \# & $\%$ \\
\hline Área de Sierras & - & - & 2 & 2.0 & 2 & 2.0 \\
\hline Barandas & 3 & 3.1 & 4 & 4.1 & 7 & 7.2 \\
\hline VMQ & - & - & 7 & 7.1 & 7 & 7.1 \\
\hline VTA & - & - & 7 & 7.1 & 7 & 7.1 \\
\hline Accesorios & 2 & 2.0 & 5 & 5.1 & 7 & 7.1 \\
\hline Mantenimiento & 1 & 1.1 & 9 & 9.2 & 10 & 10.3 \\
\hline Dpto. Técnico & - & - & 3 & 3.1 & 3 & 3.1 \\
\hline Puerta & - & - & 13 & 13.3 & 13 & 13.3 \\
\hline Recepción & - & - & 3 & 3.1 & 3 & 3.1 \\
\hline Oficinas & 5 & 5.1 & 7 & 7.1 & 12 & 12.2 \\
\hline Almacén de Ferretería & - & - & 2 & 2.0 & 2 & 2.0 \\
\hline Planta de Anodizado & 2 & 2.0 & 9 & 9.2 & 11 & 11.2 \\
\hline Cocina-Comedor & 2 & 2.0 & 4 & 4.1 & 6 & 6.1 \\
\hline Estibadores & 6 & 6.2 & 2 & 2.0 & 8 & 8.2 \\
\hline TOTAL & 21 & 21.5 & 77 & 78.5 & 98 & 100.0 \\
\hline
\end{tabular}

Fuente: Registro de Consulta de Enfermedades Profesionales

La Tabla VII fue confeccionada relacionando el resultado del audiograma con el tiempo de exposición al ruido; así podemos observar que los trabajadores más afectados fueron los que llevaban laborando entre 6 y 10 años $(23,5 \%)$, le siguen en orden de frecuencia los expuestos entre 16 y 20 años $(18,4 \%)$, lo cual se corresponde con los datos obtenidos en la literatura universal.
El Gráfico II representa la distribución de la muestra de acuerdo al diagnóstico donde observamos que del total afectado un $9,5 \%$ fue considerado como enfermedad del trabajo por encontrarse innecesariamente expuestos a ruido en sus puestos de trabajo. El 90,5\% fue diagnosticado como enfermedad profesional. 
Tabla V. Resultados del audiograma por grupos de edades

\begin{tabular}{|c|c|c|c|c|c|c|}
\hline \multirow{2}{*}{$\begin{array}{c}\text { GRUPOS DE } \\
\text { EDADES }\end{array}$} & \multicolumn{2}{|c|}{ RESULTADOS AUDIOGRAMA } & \multicolumn{2}{c|}{ TOTAL } \\
\cline { 2 - 7 } & \multicolumn{2}{|c|}{ NORMAL } & \multicolumn{2}{|c|}{ HIPOACUSIA } & \multicolumn{1}{c|}{} \\
\cline { 2 - 7 } & $\#$ & $\%$ & $\#$ & $\%$ & $\#$ & $\%$ \\
\hline 25 años o menos & 3 & 3.1 & 4 & 4.1 & 7 & 7.2 \\
\hline 26-35 años & 12 & 12.2 & 27 & 27.6 & 39 & 39.8 \\
\hline 36-45 años & 3 & 3.1 & 19 & 19.3 & 22 & 22.4 \\
\hline 46-55 años & 3 & 3.1 & 19 & 19.3 & 22 & 22.4 \\
\hline 56 años o más & - & - & 8 & 8.2 & 8 & 8.2 \\
\hline TOTAL & 21 & 21.5 & 77 & 78.5 & 98 & 100.0 \\
\hline
\end{tabular}

Fuente: Registro de Consulta de Enfermedades Profesionales

Tabla VI. Resultados del audiograma por sexos

\begin{tabular}{|c|c|c|c|c|c|c|}
\hline \multirow{2}{*}{ SEXO } & \multicolumn{3}{|c|}{ RESULTADOS AUDIOGRAMA } & \multicolumn{2}{c|}{ TOTAL } \\
\cline { 2 - 7 } & \multicolumn{2}{|c|}{ NORMAL } & \multicolumn{2}{c|}{ HIPOACUSIA } & \multicolumn{2}{|c|}{} \\
\cline { 2 - 7 } & $\#$ & $\%$ & $\#$ & $\%$ & $\#$ & $\%$ \\
\hline MASCULINO & 12 & 12.2 & 32 & 32.7 & 44 & 44.9 \\
\hline FEMENINO & 9 & 9.2 & 45 & 45.9 & 54 & 55.1 \\
\hline TOTAL & 21 & 21.4 & 77 & 78.6 & 98 & 100.0 \\
\hline
\end{tabular}

Fuente: Registro de Consulta de Enfermedades Profesionales

Tabla VII. Resultados del audiograma por tiempo de exposición

\begin{tabular}{|c|c|c|c|c|c|c|}
\hline \multirow{2}{*}{$\begin{array}{c}\text { TIEMPO } \\
\text { DE } \\
\text { EXPOSICION }\end{array}$} & \multicolumn{3}{|c|}{ RESULTADOS AUDIOGRAMA } & \multicolumn{2}{c|}{ TOTAL } \\
\cline { 2 - 7 } & \multicolumn{2}{|c|}{ NORMAL } & \multicolumn{2}{c|}{ HIPOACUSIA } & \multicolumn{2}{c|}{} \\
\cline { 2 - 7 } & $\#$ & $\%$ & $\#$ & $\%$ & $\#$ & $\%$ \\
\hline MENOS 1 ANO & 4 & 4.1 & - & - & 4 & 4.1 \\
\hline 1-5 ANNOS & 12 & 12.2 & 15 & 15.3 & 27 & 27.5 \\
\hline 6-10 ANNOS & 4 & 4.1 & 23 & 23.5 & 27 & 27.5 \\
\hline 11-15 ANNOS & - & - & 9 & 9.1 & 9 & 9.1 \\
\hline 16-20 ANNOS & - & - & 18 & 18.4 & 18 & 18.4 \\
\hline 21-30 ANNOS & 1 & 1.0 & 8 & 8.2 & 9 & 9.2 \\
\hline $\begin{array}{c}\text { 31 ANNOS O } \\
\text { MAS }\end{array}$ & - & - & 4 & 4.1 & 4 & 4.1 \\
\hline TOTAL & 21 & 21.4 & 77 & 78.6 & 98 & 100.0 \\
\hline
\end{tabular}

Fuente: Registro de Consulta de Enfermedades Profesionales 


\section{Gráfico 2. Distribución de la muestra según diagnóstico}
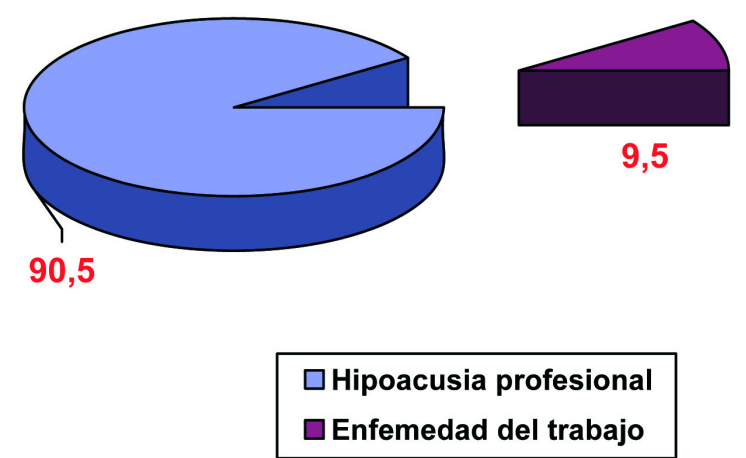

\section{DISCUSION}

El ruido industrial constituye un contaminante de gran interés que puede influir de forma negativa en la salud de los obreros expuestos al mismo en su centro laboral, por lo que muchos especialistas han dedicado su tiempo al estudio de las afectaciones auditivas condicionadas por la exposición del ambiente ruidoso por largos años sin el uso adecuado de los medios de protección.

López González L. ${ }^{17}$ en su investigación con jóvenes trabajadores de una industria textil en Cuba encontró que los obreros que utilizaban los medios de protección auditiva presentaban menos afectación que los que no lo hacían, influyendo también el tiempo de uso de los mismos. ${ }^{23}$ Otro trabajo revisado 23 acerca de los equipos de protección individual plantea que el simple hecho de retirarlos durante 5 minutos en el ambiente ruidoso de la jornada laboral hace que se diluya y desaparezca su eficacia. De ahí, la gran importancia que reviste el uso de los medios de protección auditiva para evitar daños en la salud de los trabajadores ${ }^{17.22 .23}$.

López Muñoz ${ }^{37}$ en su estudio acerca de la lucha contra el ruido en el lugar de trabajo plantea que los fabricantes se darán cuenta que reduciendo el nivel de ruido emitido por sus máquinas aumentarán sus ventas y provocarían menos afectación para el hombre.

Tosal Suárez y colaboradores ${ }^{22}$ efectuaron mediciones de los niveles de ruido en 1277 tareas en una industria española de aserrado y preparación de la madera encontrando que el nivel predominante era también superior a los $90 \mathrm{db}(\mathrm{A})$ afectando al 49,27\% de los trabajadores. Además comprobaron que no existían cabinas de aislamiento para las máquinas de corte e instalaciones auxiliares donde los niveles de ruido sobrepasaban los $90 \mathrm{db}(\mathrm{A})$.

Sánchez Ferrero M. F. ${ }^{33}$ en un estudio de 150 pacientes al azar de industria de Puertollano halló un nivel de ruido de más de $100 \mathrm{db}(\mathrm{A})$ en una jornada laboral, lo cual provocó efectos negativos en la salud de los expuestos al riesgo.

Espinosa Lozano y colaboradores ${ }^{33}$ en su estudio "El ruido industrial como patología laboral" obtuvieron gran incidencia de hipoacusia profesional como resultado del ambiente laboral ruidoso.

Similares resultados encontraron otros de los autores consultados en la revisión bibliográfica como Talbott-E.O. y colaboradores ${ }^{27}$, Tosal Suárez J. M. 22 y Monasterio Acha R. ${ }^{29}$. Este último realizó un estudio de 746 pacientes que laboraban en astilleros del Nervión S.A. encontrando que el $65,9 \%$ presentaban hipoacusia en mayor o menos grado, pero solo el $1,33 \%$ fue considerada como enfermedad profesional. ${ }^{15.22 .24 .25 .26 .27 .28 .29 .33 . ~}$

Talbott-E.O. y colaboradores ${ }^{27}$ en un estudio de 245 obreros jubilados con edades comprendidas entre 56 y 68 años de 30 años encontraron a través de los test audiométricos indicados severos daños en la audición del $67 \%$ de los más viejos (edades entre 64 y 68 años).

Otros trabajos realizados coinciden con nuestros resultados, puesto que la hipoacusia ocupacional se manifestará con mayor frecuencia en los oídos de los más viejos que en los oídos de los más jóvenes. ${ }^{31.32}$.

Calviño del Río A. y colaboradores ${ }^{25}$ en un trabajo presentado en la Revista Cubana de Higiene y Epidemiología concluyeron que las mujeres eran 
menos sensibles a las caídas del umbral auditivo, lo cual difiere de nuestros resultados.

Otros autores señalan que es muy importante considerar también el sexo ya que para la misma edad la capacidad auditiva de las mujeres es más pobre y vulnerable que en los hombres, lo cual si coincide con los hallazgos de nuestro trabajo ${ }^{24.31}$.

En la Enciclopedia de Medicina y Seguridad del Trabajo aparece reflejado que la intermitencia en la exposición tiende a reducir los efectos negativos del ruido peligroso; no obstante, cuanto más larga sea la duración de la exposición mayor es el riesgo ${ }^{1}$.

Clemente Ibáñez ${ }^{8}$ planteó en una investigación publicada en la Revista de Medicina y Seguridad del Trabajo de Madrid que el efecto nocivo del ruido es proporcional a la duración de la exposición al mismo.

López González L. ${ }^{17}$ en un estudio realizado a 88 trabajadores del Combinado Textil "Desembarco del Granma" con más de 8 meses vinculados al trabajo concluyó que la polución acústica existente había incidido negativamente, a pesar del poco tiempo de exposición, en la salud del personal expuesto provocando alteraciones auditivas.

Calviño del Río A. y otros ${ }^{25}$ comprobaron la relación directa entre los altos niveles de ruido y muchos años de exposición, con la incidencia de hipoacusia profesional.

Cerrada Delgado M. C. ${ }^{32}$ en un estudio de 120 obreros de menos de 50 años de edad de un astillero encontró un porciento elevado de pérdida de la audición relacionada con los puestos de trabajo más ruidosos del taller y demostró que guardaba relación con los años de exposición al ruido industrial.

Guerrero Pupo. J. C. ${ }^{24}$ en su análisis con obrero de la fábrica de combinadas cañeras KTP de Holguín demostró la existencia de un $40,9 \%$ de hipoacusia ocupacional en 5 de los talleres con que cuenta la instalación.

La sordera ocupacional como consecuencia de la exposición prolongada al excesivo ruido industrial es una enfermedad que ha sido objeto de estudio de muchos de los trabajos revisados, los cuales coinciden con nuestros resultados al obtener elevados porcientos de afectados por esta causa como ya expusimos anteriormente 22.24 .29 .33 .35 .

\section{CONCLUSIONES}

1. En esta industria el ruido constituye un contaminante de gran importancia. Este riesgo laboral se encontró por encima del nivel de seguridad de $85 \mathrm{db}(\mathrm{A})$ en 9 de los 13 departamentos con que cuenta el centro.

2. Los departamentos donde mayores niveles sonoros se detectaron fueron: Área de Sierras, Baranda, V.T.A. (Tablillas), V.M.Q. (Marquesinas) y Accesorios, los cuales sobrepasaron los $90 \mathrm{db}(\mathrm{A})$.

3. El ruido ha afectado la salud de los trabajadores de este centro, ya que se encontraron 77 casos de hipoacusia atribuible al ruido, lo que representó el 78,5\% del total estudiado.

4. Existía un gran número de trabajadores $(30,6 \%)$ expuestos elevados niveles de ruido innecesariamente por la naturaleza de su labor.

\section{RECOMENDACIONES}

1. Deben tomarse medidas que reduzcan el ruido en los puestos de trabajo donde existen niveles superiores a los $85 \mathrm{db}(\mathrm{A})$, mediante casetas u otras instalaciones similares donde el trabajador pueda permanecer sin estar expuesto a altos niveles sonoros.

2. Debe exigirse y controlarse el uso adecuado de los medios de protección auditiva en los trabajadores con exposición elevada al ruido.

3. Se hace necesario el estricto cumplimiento de los Exámenes Médicos Preventivos a los trabajadores de esta industria, incluyendo las pruebas audiométricas anualmente. Es indispensable la educación sanitaria del personal administrativo y de los obreros. 


\section{BIBLIOGRAFIA}

1. Enciclopedia de Medicina, Higiene y Seguridad del Trabajo. Volumen II. Instituto Nacional de Medicina y Seguridad del Trabajo. Editorial de Revadeneyre, S.A., Madrid, 1979.

2. Legislación en materia de ruido y criterios de valoración del ruido. Instituto Tecnológico de Seguridad MAPFRE (ITSEMAP), Madrid, 1988.

3. Escobar, H. Ruidos en Cuba, MINSAP. Curso de Medicina del Trabajo. Editorial Orbe, 1978. p. 103-107.

4. Morón, A.G. Otorrinolaringología clínica. Editorial Espaxs, Barcelona, 1981. p. 137.

5. Higiene del Medio. La Habana, Dirección Nacional de Higiene, Ministerio de Salud Pública, 1976. T.3.

6. NC - 19-01-04 de 1980. Ruido: Requisitos generales higiénicos-sanitarios.

7. Rodríguez L. Manual de Medicina del Trabajo, Editorial Pueblo y Educación, 1989.

8. Clemente Ibáñez, M. Enfermedades profesionales del oído. Revista Medicina y Seguridad del Trabajo, $\mathrm{N}^{\circ}$ 152, Abril-Junio, 1991.

9. Andlauer, P. La enfermedad profesional y la vigilancia especial en: El ejercicio de la Medicina del Trabajo. Barcelona, Editorial Científico-Médica, 1980.

10. Fernández Orozco, F. Enfermedades producidas por ruido. Gaceta Médica México, 114 (9): 416-19, Septiembre, 1978.

11. Pareja, H. El ruido un mal de nuestro tiempo. Revista Consulta Médica, No 246, 1984, p. 4-5.

12. Urbán, L.M. Efectos sobre la salud de los trabajadores expuestos a ruido. Trabajo final. Curso de Maestría Internacional en Salud Ocupacional. C. Habana, 1985.

13. Alvarez Alvarez, A. Otorrinolaringología ocupacional. Conferencia impartida. Centro Provincial de Higiene-Epidemiología, C. Habana, 1985.

14. Comellas C. y otros. Riesgos a la exposición al ruido en la industria azucarera. Revista Cubana HigieneEpidemiología, 20:2, Abril-Junio, 1982.

15. Barceló, C. Física del sonido: Conferencia Instituto Nacional Higiene-Epidemiología, 1984, p. 1, 12, 25,27.

16. Carvajal, L. Evaluación de los riesgos físicos y químicos presentes en el ambiente laboral del personal médico y paramédico del Hospital "Hermanos Ameijeiras". Tesis especialista 1er. Grado en Medicina del Trabajo, C. Habana, 1988.

17. López González, L. Comportamiento del umbral auditivo en jóvenes trabajadores de una industria textil., Tesis especialista 1er. Grado en Medicina del Trabajo, C. Habana. 1989.
18. NC 19-01-13 de 1983. Ruido: Determinación de la pérdida de la audición.

19. ILO: Occupational Health and Safety, Geneve, 1971.

20. González, J.P. El ruido dañando a la audición. Instituto de Medicina del Trabajo. C. Habana, 1985. Comunicación personal.

21. Introducción a la acústica. Instituto Tecnológico de Seguridad MAPFRE (ITSEMAP). Conferencia del 1er Curso Master en Seguridad Integral. Madrid, 1988.

22. Tosal, J.M. y Santa María, G. Riesgo profesional en aserrado y preparación industria de la madera. Revista Salud y Trabajo. Madrid, No 89, Enero, 1992.

23. Cárcoba, A.C. Los equipos de protección individual. Revista Salud y Trabajo. Madrid, No 90, Febrero, 1992.

24. Guerrero, J.C, El ruido como agente agresor a la salud en la fábrica de combinadas cañeras KTP de Holguín. Tesis especialista 1er. Grado en Medicina del Trabajo. C. Habana, 1985.

25. Calviño del Río, A. y otros. La sordera profesional: enfermedad frecuente en la práctica de la salud ocupacional. Informe preliminar. Revista Cubana Higiene-Epidemiología, 20(3):408, 1982.

26. Lucchini, R. et al. Effects on hearing in exposure to impulsive and high frequency noise. Med-Lavoro 82(6):547-53, November-December, 1991.

27. Talbott-ED, et al. Noise induced hearing loss: a possible marker for high blood pressure in older noise exposed populations, J. Occup-Med 32(8): 690-1, August, 1990.

28. Lalande-NM, et al. Quantification of the psychosocial disadvantages experienced by workers in a noisy industry and their nearest relatives: perspectives for rehabilitation. Audiology 27(4): 196-206, 1988.

29. Monasterio, R. y Serrano, M.B. Patología del ruido. Revista Medicina y Seguridad del Trabajo, $N^{\circ} 152$, Abril-Junio, 1991.

30. Cabrera, E.F. Susceptibilidad al ruido en trabajadores de la industria textil. Tesis especialista 1er grado en Medicina del Trabajo, C. Habana, 1986.

31. Mc Shane D.P., et al. Unilateral otosclerosis and noise-induced occupational hearing loss. Clin. Otolaryngology 16(1): 70-5, February, 1991.

32. Cerrada Delgado, M.C. Efecto sobre la audición en ambiente laboral ruidoso. Revista Medicina y Seguridad del Trabajo, Madrid, N 152, Abril-Junio, 1991.

33. Espinosa, F.J. y Sánchez, M.F. El ruido industrial 
como patología laboral. Revista Medicina y Seguridad del Trabajo, Madrid, No 152, Abril-Junio, 1991.

34. Sineva-E.L. et al. Early signs of the effect of complex occupational factors of plasma technology on the worker's health status. Gig - Tr - Prof - Zabol. 23-5, March, 1991.

35. Vilas, J. Curso general de Medicina del Trabajo. Enfermedades profesionales por agentes físicos: Ruido. Instituto Nacional Seguridad e Higiene del Trabajo. Pamplona, 1985.
36. Castejón, E. La contaminación del medio ambiente de trabajo. Revista Salud y Trabajo. INSHT, Madrid, N $^{\circ}$ 89, Enero, 1992.

37. López Muñoz, G. Una visión actual de la estrategia comunitaria en la lucha contra el ruido en el lugar de trabajo. Revista Salud y Trabajo, INSHT, Madrid, No 90. Febrero, 1992.

38. Resolución $\mathrm{N}^{\mathrm{o}} 34$ del $1^{\circ}$ de agosto de 1977 (CETSS). 
